\title{
Sensing UMTS Bands Using Cyclostationary Features and Cooperation Between Opportunistic Terminals
}

\author{
Paulo Marques \\ Escola Superior de Tecnologia \\ Instituto Politécnico de Castelo Branco \\ Castelo Branco, Portugal \\ paulomarques@est.ipcb.pt
}

\author{
Joaquim Bastos, Atílio Gameiro \\ Instituto de Telecomunicações \\ Universidade de Aveiro \\ Aveiro, Portugal \\ jbastos@av.it.pt,amg@ua.pt
}

\begin{abstract}
The Opportunistic Radio (OR) concept relies on the cognitive features of the OR terminals, namely the ability to adapt its transmitter parameters, based upon interaction with the RF environment in which it operates. An OR system operates in licensed frequency bands, exploiting opportunities and operating with a lower priority regarding the licensed system, implementing a spectrum pool mechanism. The most important constraint is that the OR network should always avoid harmful interference with the licensed system, therefore it should reliably detect licensed signals in the used band in order to avoid interfering with the licensed owner of that band. Given the importance of UMTS systems in current wireless communications, this paper is focused on 3G bands and addresses the problem of sensing weak UMTS signals. The proposed sensing algorithm exploits the cyclostationary features of UMTS signals and the cooperation between multiple OR terminals clustered in the OR network.
\end{abstract}

Keywords-Opportunistic Radio, Cognitive Radio, Cyclostationarity, Cooperative Sensing, UMTS Signals Sensing

\section{INTRODUCTION}

Radio spectrum has become a very expensive commodity. The opportunistic use of some frequency bands could be one way for the owners of those licensed bands to capitalize some extra revenue. As a basic principle, the Opportunistic Radio node rents the most appropriate frequency band to transmit the required power [1]. When required, an OR terminal sweeps the licensed frequency bands in order to detect spectrum holes, and adapts its transmission characteristics in order to take advantage of its spectrum sensing findings.

The UMTS system is based on CDMA, thus all users transmit at the same time, information spreaded over $5 \mathrm{MHz}$ bandwidth and therefore users interfere with each other. Signal transmission in UMTS is based on direct sequence spread spectrum (DS-SS) techniques, which poses additional challenges for detecting those signals in the RF spectrum. Since the power is spread over a bandwidth significantly higher than the one needed for a nonspread signal scheme, efficient detection techniques ensuring a very small probability of missed detection and, in this way, to avoid the "hidden terminal" problem [2], will have to explore signal features of the spread signals.

It is clear that a very reliable sensing algorithm will be necessary to avoid causing any harmful interference to the UMTS network. For illustrative purposes, considering a UMTS voice service with power efficiency target $\left(E_{b} / N_{0}\right)$ of $9 \mathrm{~dB}$ and a spreading factor $(S F)$ of 16 , the SNR target at the UMTS terminal is $\mathrm{SNR}=(1 / S F)\left(E_{b} / N_{0}\right)=-16 \mathrm{~dB}$.

This communication is organized as follows: In Section 2 the considered cooperative sensing scenario is described, in Section 3 we detail the proposed local sensing algorithm, the cyclostationary detector, while in Section 4 we present a cooperative extension of that sensing algorithm. Section 5 refers to results achieved with the presented algorithms, where also some performance comparisons are made, and in Section 6 we present the most important conclusions of this work.

\section{SCENARIO DEFINITION}

Fig. 1 illustrates a scenario with $\mathrm{N}$ ORs, grouped in a cooperation cluster, and two considered UMTS DL bands, f1 and $\mathrm{f} 2$. Band $\mathrm{fl}$ is in use (hypothesis $\mathrm{H} 1$ ) and band $\mathrm{f} 2$ is not being used by any UMTS operator in that area (hypothesis H0). We set the threshold level equal to $0.15 \mathrm{~dB}$ and the observation time equal to $30 \mathrm{~ms}$. Because of the shadowing effect, OR1 is not able to detect the UMTS signal being transmitted in the fl band and simultaneously does a false alarm decision regarding f2, consequently, OR1 starts transmitting in f1 band causing harmful interference with the neighbour UMTS licensed terminal. We can say that OR1 is affected by the hidden terminal problem [2]. Concerning OR3, it detects UMTS activity in the $\mathrm{fl}$ band and the opportunity in $\mathrm{f} 2$, thus it can start transmitting using the opportunity in the $f 2$ band. Finally, OR2 cannot transmit because it does a false alarm decision regarding the $\mathrm{f} 2$ band and correctly detects UMTS signal activity in the f1 band.

The detection statistics given by each OR node in the cluster can be shared as represented in Fig. 1, creating a cooperative scenario. We assume that the $N$ OR nodes are clustered in a circular area with radius $d$, and the centre of that 


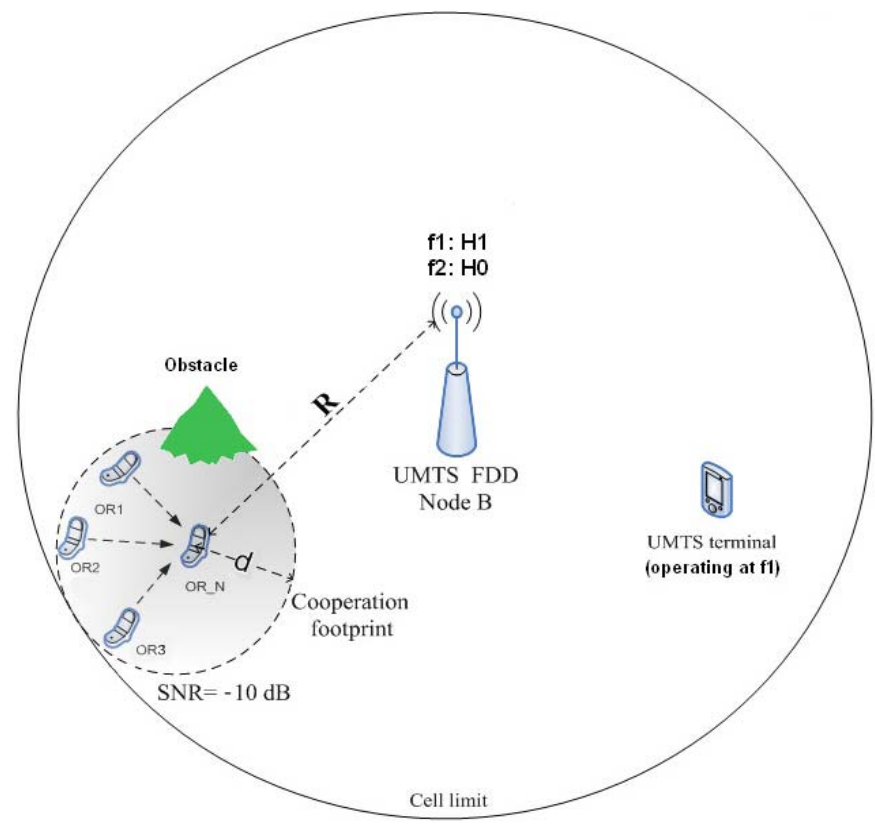

Figure 1. Cooperative sensing scenario

circle is at distance $R$ from the UMTS Node B. It is also considered that the area of the OR network is small when compared against the area of the UMTS cell $(d<R)$, thus the average SNR within the cooperation footprint is considered to be approximately the same, i.e. they are located relatively close to each other so we can assume that. Every OR clustered in the cooperation footprint sends its sensing decision to a designated central sensing decision unit (CSDU) entity. This central entity can be one of the OR nodes in the cooperation cluster.

\section{LOCAL SENSING AlgORITHM}

DS-CDMA signals can be detected exploiting the baseband cyclostationary properties that come from the redundancy between frequency components separated by multiples of the symbol rate, i.e. the cyclic feature appears at $\alpha=1 /\left(S F . T_{c}\right)$, where $S F$ is the spreading factor and $T_{c}$ is the chip duration. However, UMTS FDD standard employs, in addition to user specific spreading, the so called scrambling sequences in order to improve the correlation characteristics of the signals and provide base station identification [3]. Scrambling takes place over multiple symbols, with period equal to $10 \mathrm{~ms}$, removing the cyclostationarity with the symbol rate. Nevertheless in the UMTS standard, users' signals have always the same chip rate, even if the individual $S F$ and symbol rates differ. Thus $\alpha_{c}=1 / T_{c}$ (3.84 Mchip/s) is a common cyclic frequency to all downlink signals and the most appropriate to detect the received signal. An analytical formulation of the cyclic autocorrelation function for a UMTS FDD signal at $\alpha_{c}=1 / T_{c}$ can be found in [4]. In this approach we assume that the OR knows a priori the UMTS carrier frequencies and bandwidths, which have been brought to the baseband.

Cyclostationary signal analysis offers the ability to detect and classify signals with levels of performance approaching those of optimal coherent schemes, without needing the phase information required by those approaches, maintaining the generality of other noncoherent approaches while overcoming their main limitations.

Man-made modulated signals are in general coupled with sine wave carriers, pulse trains, spreading codes, cyclic prefixes, etc., which results in built-in periodicity. Even though in digital communications data symbol sequences may be in a large number of cases characterized as a discrete stationary random process, the continuous modulated signals are characterized as cyclostationary since their statistics, mean and autocorrelation, exhibit periodicity. This periodicity is typically a feature of man-made signals and therefore a receiver or sensor can exploit it for detection of random signals with a particular modulation type in a background of noise and other modulated signals.

The most common analysis tools for stationary random signals rely on the second order statistics, i.e. are based on the autocorrelation function and the PSD, and it is well known that for such signals nonoverlapping frequency bands are uncorrelated. On the other hand, the periodicity inherent in cyclostationary signals implies some spectral redundancy which results in correlation between non-overlapping spectral components separated by some multiple of cycles [5]. In analogy with the definition of conventional autocorrelation, one can define for each cycle $\alpha$ of a cyclostationary signal a spectral correlation function

$$
R_{x}(\tau, \alpha)=\lim _{\text {Tobs } \rightarrow \infty} \frac{1}{T o b s} \int_{-T o b s / 2}^{\text {Tobs } / 2} x\left(t+\frac{\tau}{2}\right) x *\left(t-\frac{\tau}{2}\right) e^{-j 2 \pi \alpha t} d t,
$$

where $x$ is the signal of interest and the new parameter $\alpha$ is known as the cycle frequency. Tobs is the observation time spent detecting the signal. When $\alpha$ is set to zero, we obtain the particular case of the conventional energy detector, and for stationary signals $R_{x}(\tau, \alpha)=0, \forall \alpha \neq 0$. The Spectrum Cyclic Density (SCD) is a Fourier transform of the cyclic autocorrelation, given by

$$
S_{x}(f, \alpha)=\int_{-\infty}^{\infty} R_{x}(\tau, \alpha) e^{-j 2 \pi f \tau} d \tau .
$$

If the $x$ signal and noise are uncorrelated, the SCD of the received signal $y$ is given by

$$
S_{y}(f, \alpha)=S_{x}(f, \alpha)+S_{n}(f, \alpha) .
$$

Assuming that the additive noise, $n$, is as a stationary process, then its SCD tends to zero for $\alpha \neq 0$ as Tobs is made infinitely large

$$
\lim _{\text {Tobs } \rightarrow \infty} S_{n}(f)=0, \quad \forall \alpha \neq 0 .
$$

Unlike the PSD which is a real-valued one dimensional transform, the SCD is a two dimensional transform. The distinctive characteristic of spectral redundancy makes signal selectivity possible, thus, overlapping features in the power spectrum density are not overlapping features in the cyclic spectrum, i.e. in the $\alpha$ domain.

The output of the feature detector is the detection statistic $d$, which is a metric representing the amount of spectral correlation present in the received signal. For the single 
spectral line in $\alpha$ domain, in order to obtain the optimal detection statistic we should maximize the detected power computing $Z_{\text {opt }}$ given by

$$
Z_{\text {opt }}=\int_{-f_{s} / 2}^{f_{s} / 2} S_{y}\left(f, \alpha_{0}\right) S_{x}^{*}\left(f, \alpha_{0}\right) d f,
$$

where $f_{S}$ is the sampling frequency, $S_{y}$ is the SCD of the received signal $y$, and $S_{x}$ is the SCD of the signal of interest, $x$. However, the optimal detector cannot be implemented without knowledge of the transmitted UMTS signals' phase because $S_{y}$ depends on this phase. Our proposal is to use a sub-optimal approach without requiring phase-related information given by

$$
Z=\int_{-f s / 2}^{f_{s} / 2}\left|S_{y}\left(f, \alpha_{0}\right)\right|^{2} d f
$$

We have chosen as detection statistic the SNR measured at a single spectral line. In practical implementations of the cyclostationarity detector the Tobs is limited, therefore the theoretical limit given by (4) cannot be reached and there will always be a noise floor of $S_{y}>0$, constant for all $\alpha \neq 0$. In order to estimate this noise floor we take measurements of the noise at any cyclic frequency, $\alpha_{n}$, where it is guaranteed to be no cyclic features present, i.e. $\alpha_{\mathrm{n}} \neq \alpha_{0}$, using any frequency other than the $\alpha_{0}$ single spectral line. Therefore,

$$
Z_{n}=\int_{-f_{s} / 2}^{f_{s} / 2}\left|S_{y}\left(f, \alpha_{n}\right)\right|^{2} d f \quad, \quad \alpha_{n} \neq \alpha_{0},
$$

and the detection statistic at the single $\alpha_{0}$ cyclic frequency is given by

$$
d=10 \log \left(\frac{Z}{Z_{n}}\right) .
$$

Consequently, the detection statistic $d$ tends to $0 \mathrm{~dB}$ when $Z=Z_{n}$, i.e. the signal is absent. Usually the cyclostationary detector exploits the fundamental cyclic frequency, i.e. $\alpha=\alpha_{0}$.

Fig. 2 is the cyclostationary detector block diagram, using a periodogram approach. After an FFT operation a sliding window of samples performs frequency shifts of $+\alpha / 2$ and $-\alpha / 2$. The shifted spectrums are then multiplied to obtain the Spectrum Cyclic Density function (SCD). After that, a time smoothing operation is done using an average over $K$ sets of $N$ samples, during the observation time. The complex values are then squared and integrated over the $f$ domain. Finally, the detection statistic $d$ is given by the ratio between the power of the cyclostationary feature, measured at cyclic frequency $\alpha_{c}$,

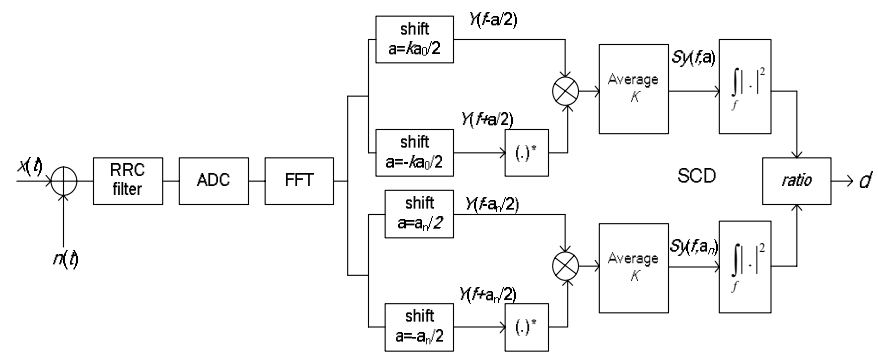

Figure 2. Cyclostationary detector for UMTS signals (local sensing) and the estimated noise floor, measured at $\alpha_{n}$. In order to estimate this noise floor we take measurements of the noise at any cyclic frequency $\alpha_{n}$, where it is guaranteed to be no cyclic features present. Notice that since the UMTS chip rate is a standard frequency there is no need to search over the SCD function, keeping the detector in a low complexity level.

The SCD of a UMTS FDD signal is shown in Fig. 3 for an $\mathrm{SNR}=-5 \mathrm{~dB}$. For that SNR level, the cyclostationary features vanish due to the effect of cross-spectral correlation between the signals and noise but still have a visible pattern that will allow signal detection.

It is important to notice that feature detection is compromised when the UMTS signal experiences multipath propagation, which can cause deep fading in some signal subbands, loss of correlation between frequencies and consequently degradation of the cyclostationary features intended to detect.

\section{COOPERATIVE EXTENSION OF UMTS SIGNAL DETECTOR}

A network of ORs that can exchange detection decisions, among each other or with a centralized entity, can be quite rewarding regarding detection performance in some particular scenarios, namely in the well known hidden terminal problem. Whenever an OR experiences shadowing or fading effects, the detection of an active licensed user transmitting is compromised, and in such cases the OR in question is unable to differentiate an unused band from a deep fade [6]. To mitigate these effects it is possible to implement a cooperative spectrum sensing scheme where the sensing information from multiple ORs is gathered before a detection decision is taken whether the sensed licensed spectrum band is free or being used by some licensed user, according to a specific decision taking algorithm which ponders all collected sensing metrics from ORs in a particular sensing cluster.

Performance analysis of the UMTS local sensing algorithm show that a single node can detect an UMTS signal with an $\mathrm{SNR}=-10 \mathrm{~dB}$ with a probability of detection $(P d)$ of approximately 1 , and a negligible probability of false alarm $(P f a)$, for a $T o b s=30 \mathrm{~ms}$. It is expected that the cooperative extension of this algorithm can maintain the same reliability while spending less observation time, which means lower complexity and processing time.

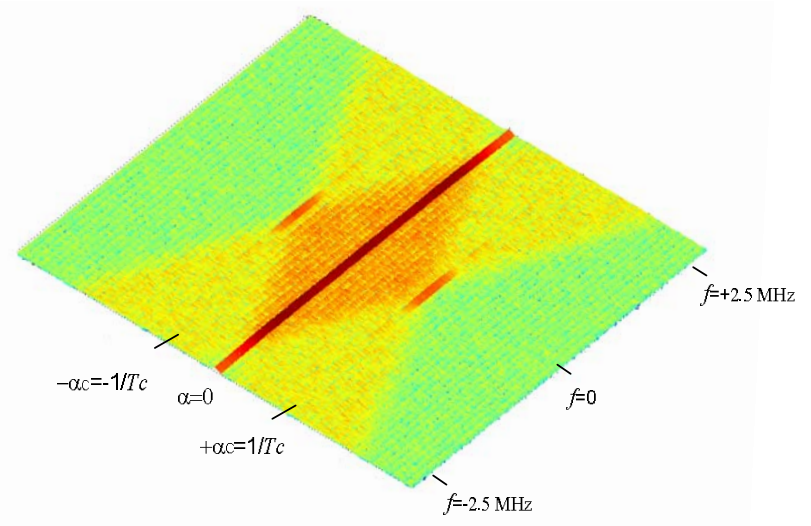

Figure 3. $\operatorname{SCD}(\alpha, \mathrm{f})$ function of UMTS FDD signal $($ Tobs $=100 \mathrm{~ms}, \mathrm{SNR}=-5 \mathrm{~dB})$ 
We can classify decision rules in two kinds: hard decision and soft decision. In hard decision rules only local decisions ( $\mathrm{H} 0$ or $\mathrm{H1}$ ) are shared within the OR network, while in soft decision rules statistic information coming from different OR nodes, e.g. the value of $d$, is shared between the ORs. Previous research work concluded that hard decision rules achieve cooperative gain values nearly identical to soft decision ones [7]. In addition, we expect a low bandwidth control channel, thus it is realistic to assume that radios exchange hard decisions $(\mathrm{H} 0$ or $\mathrm{H} 1)$ rather than statistics or long vectors of raw data.

In this scenario we use an "or" fusion rule for the reason that given a targeted probability of detection $P d c$, or a targeted probability of false alarm $P f a c$, the individual opportunistic nodes' threshold can be easily derived. In "or" fusion rule, when at least one out of $N$ ORs detects the UMTS signal, the resulting cooperative decision is that the UMTS signal is present, meaning the decision is $\mathrm{H} 1$ if any of the $N$ local decisions is $\mathrm{H} 1$.

In independent channel assumption, the cooperative rule combines independent measurements, thus a probability of detection of the cooperation scheme $(P d c)$ monotonically increases as

$$
P d c=1-(1-P d)^{N} .
$$

However, the probability of false alarm for the cooperation scheme (Pfac), also monotonically increases as

$$
P f a c=1-(1-P f a)^{N} .
$$

\section{Results}

Simulation results, considering an AWGN channel, show that a single OR terminal can detect an UMTS signal with an SNR of $-10 \mathrm{~dB}$, for a Tobs of $30 \mathrm{~ms}$, assuring a $99.9 \%$ probability of detection while having a negligible probability of false alarm, as Receiver Operating Characteristic (ROC) curves show in Fig. 4.

The channel correlation between OR nodes is an important issue when cooperative schemes are analysed. Increased

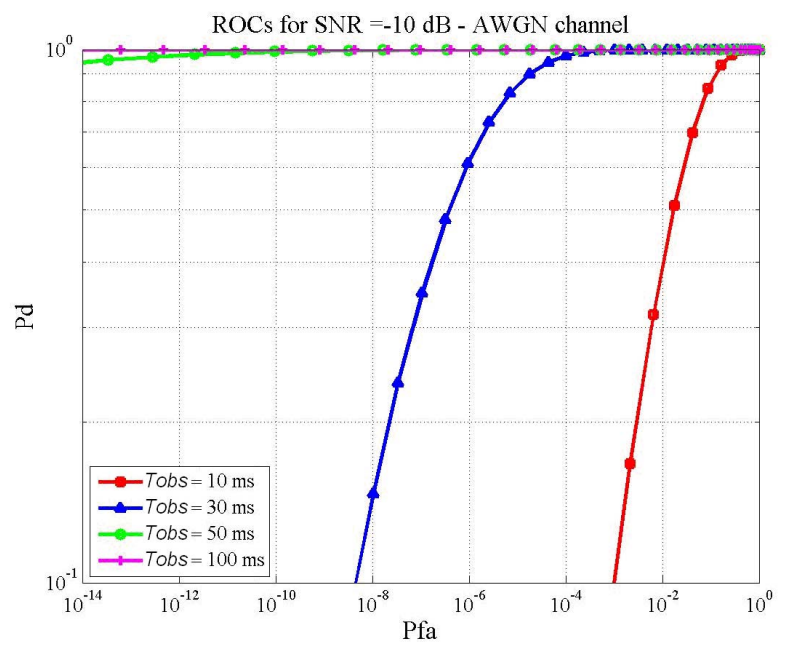

Figure 4. Cyclostationary detector ROCs for $\mathrm{SNR}=-10 \mathrm{~dB}$ (AWGN channel) correlation decreases the chance of getting an OR node with a very good channel and hence more users need to be polled for independent readings of the same signal. This correlation comes primarily from shadowing. In fact, shadowing can exhibit high correlation if two OR nodes are blocked by the same obstacle. Based on measurements reported in [8] it is reasonable to consider a shadowing model that follows a lognormal distribution, and an exponential correlation given by

$$
\rho(\Delta d)=e^{-a \Delta d},
$$

where $d$ is the distance between each of the two ORs' positions, and from measurements: $a=0.12$ (urban); $a=0.002$ (suburban). For illustrative purposes, Table 1 shows some typical values for urban and suburban scenarios.

TABLE I. TYPICAL VALUES FOR URBAN AND SUBURBAN SCENARIOS

\begin{tabular}{|c|c|c|}
\hline $\mathbf{d}[\mathbf{m}]$ & $\boldsymbol{\rho}$ urban & $\boldsymbol{\rho}$ suburban \\
\hline 10 & 0.3 & 0.98 \\
\hline 50 & 0.002 & 0.90 \\
\hline 100 & $\approx 0$ & 0.81 \\
\hline
\end{tabular}

The sensed SNR at the antenna of an OR node is defined according to

$$
S N R=S N R_{\text {nominal }}+S, \quad S \sim N(0, \sigma),
$$

where $S$ is the shadowing that follows a normal distribution with standard deviation $\sigma=5 \mathrm{~dB}$.

In order to evaluate the cooperative gain, simulations were carried out. Fig. 5 illustrates the $P d c$ and the $P f a c$, achieved with the cooperative sensing, as a function of the number of consulted OR nodes, $N$, for different correlation shadowing levels. These results were obtained for a nominal $\mathrm{SNR}=-10 \mathrm{~dB}$ within the cooperative footprint, and $T o b s=10 \mathrm{~ms}$. It is considered that every OR node spends the same observation time sensing the signal before decision. Results show that there is a monotonical increase of $P d c$ as the number of OR nodes increases. It is clear the impact of the shadowing correlation level on the cooperative sensing performance. In fact, shadowing correlation decreases the amount of new info that an

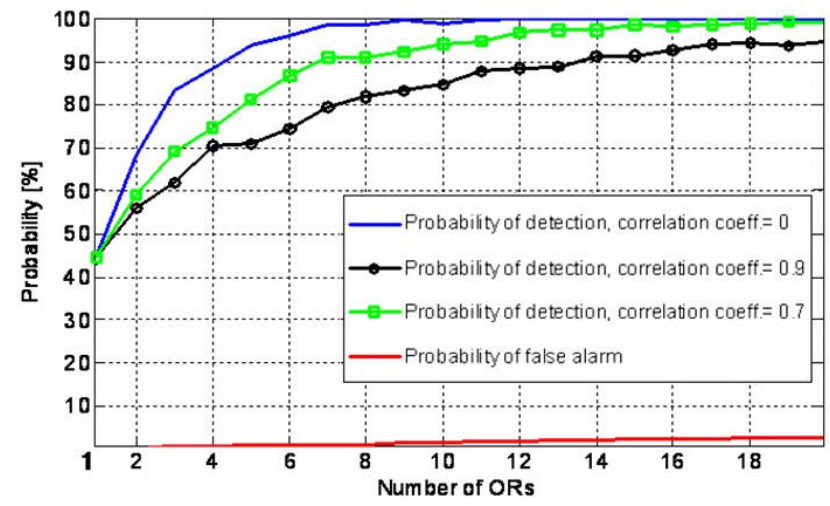

Figure 5. Pdc, $P$ fac as a function of consulted $\mathrm{ORs}(\mathrm{SNR}=-10 \mathrm{~dB}, \mathrm{Tobs}=10 \mathrm{~ms})$ 
OR node gets from other ORs, destroying part of the cooperative gain.

There is a saturation of the cooperative gain, which also depends on the shadowing, i.e. for no correlated shadowing saturation occurs for $N=10$, while for a correlation coefficient of 0.7 saturation occurs for $N=18$ nodes. Regarding the Pfac, there is also a slow increase as the number of ORs rises, varying from $(N=1,0.1 \%)$ to $(N=18,2 \%)$, however it is worthwhile since the related increase of the $P d c$ decreases the probability of the OR network to interfere with the licensed UMTS system.

Fig. 6 shows the $P d c$ as a function of the nominal SNR within the cooperative footprint for $N=1$ and $N=10$ OR nodes. The comparison between these curves, for the same $P d c$ target, shows the cooperative gain of the sensing algorithm.

\section{CONCLUSIONS}

The proposed cyclostationary feature detector exploits the cyclic frequency common to all downlink signals in a UMTS cellular system, which comes from the UMTS chip rate, assuming the OR knows the UMTS carrier frequencies and bandwidths. For that, the detector, using a periodogram approach, relies on second order statistics, based on spectrum cyclic density function. The output of the detector, after all signal processing, is a detection statistic, $d$, in $\mathrm{dB}$, which represents the ratio between the power of the cyclostationary

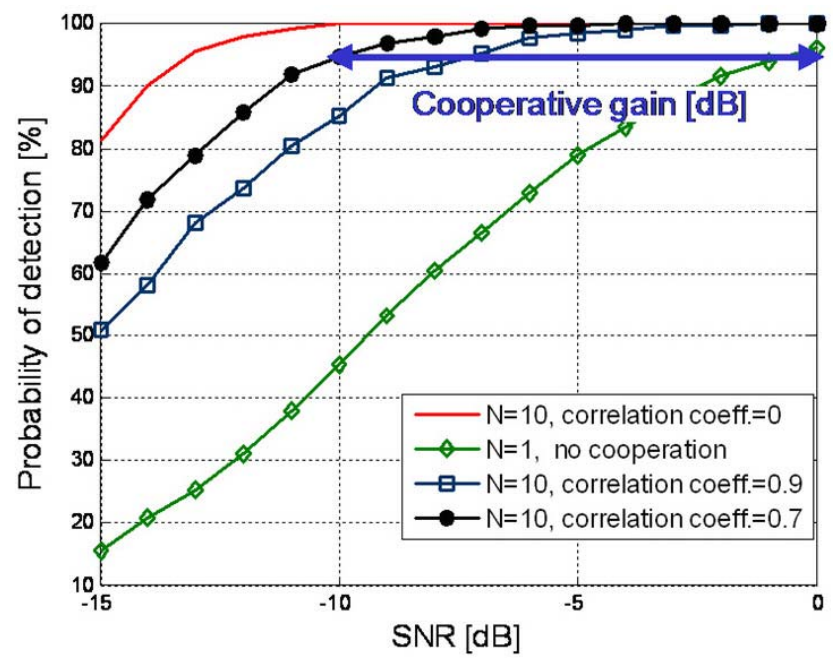

Figure 6. Pdc as a function of the nominal SNR (Tobs= $10 \mathrm{~ms}$ ) feature measured at cyclic frequency $\alpha_{c}$, and the estimated noise floor measured at $\alpha_{n}$.

A cooperative sensing scheme helps reduce the shadowing effect in the decision making process since it provides multiple independent realizations, the probability that all ORs are simultaneously faded is very low. However, shadowing correlation decreases the amount of new info that an OR gets from others, destroying part of the theoretical cooperative gain.

Results indicate a significant decrease of the average SNR required for detecting an UMTS signal when cooperation is employed. Thus, it is possible to obtain a cooperative $P d c$ of 99.9\% with an SNR of $-10 \mathrm{~dB}$, combining 10 OR nodes, each with a local $P d$ of $43 \%$, for a Tobs of $10 \mathrm{~ms}$, while a single local sensor needs $30 \mathrm{~ms}$ of Tobs to achieve that same $P d$.

Cooperative spectrum sensing allows a reduction of the sensitivity requirements at individual nodes, leading to a decrease in signal processing complexity and required observation time. Cooperative sensing can also help to overcome the hidden node problem.

\section{ACKNOWLEDGMENT}

The work presented in this paper was supported by the European project IST-ORACLE and Portuguese Foundation for Science and Technology (FCT) through project AGILE.

\section{REFERENCES}

[1] T. A. Weiss, F. K. Jondral, "Spectrum Polling: an innovative strategy for the enhancement of spectrum efficiency", IEEE Communications Magazine, vol. 42, iss. 3, Mar. 2004, pp. 8 - 14.

[2] IEEE 802.22 Working Group web site: http://www.ieee802.org/22.

[3] 3GPP Technical Specification Group Radio Access Network, "Physical channels and mapping of transport channels onto physical channels (FDD) (Release 6)", TS 25.211, v6.7.0, Dec. 2005.

[4] M. Oner, F. Jondral, "Air interface identification for Software Radio Systems", AEU International Journal of Electronics and Communication, vol. 61, iss 2, Feb. 2007, pp. 104 - 117.

[5] W. A. Gardner, Cyclostationarity in Communications and Signal Processing, IEEE Press, 1994.

[6] A. Ghasemi, E. S. Sousa, "Collaborative Spectrum Sensing for Opportunistic Access in Fading Environments", IEEE DySPAN 2005, Nov. 2005, pp. 131 - 136.

[7] S. M. Mishra, A. Sahai and R. W. Brodersen, "Cooperative Sensing among Cognitive Radios”, IEEE ICC 2006, vol. 4, June 2006, pp. 1658 -1663 .

[8] M. Gudmundson, "Correlation model for shadow fading in mobile radio systems”, Electronics Letters, vol. 27, iss. 23, Nov. 1991, pp. 2145 2146 . 